\title{
Meeting the affordability challenges posed by orphan drugs: a survey of payers, providers, and employers
}

\author{
Erin Lopata, PharmD, MPH; Christopher Terrone, MS; Ami Gopalan, PharmD, MBA, FAMCP; \\ Nicholas Ladikos, PharmD, BCPS, BCGP, BCIDP; and Terry Richardson, PharmD, BCACP
}

\section{What is already known about this subject}

- Payers and employers have traditionally managed high-cost orphan drugs through utilization management strategies, with coverage and level of restriction varying across payers and employers.

- With a robust pipeline of orphan drugs anticipated to enter the market, including gene therapies and other high-cost, 1-time treatments, many health care stakeholders are questioning if they can afford to continue to cover them for their populations.

\section{What this study adds}

- The perspectives of a diverse group of health care stakeholders, including payers, employers, and providers, on current and future strategies to manage orphan drugs.

- As providers navigate payer coverage, benefit, and site-of-care requirements in the provision of care to patients with rare conditions, they are considering the cost burden on patients as well as the administrative burden related to the acquisition of orphan drugs.

\section{ABSTRACT}

BACKGROUND: As an increasing number of orphan drugs are FDA approved, health care payers, employers, and providers are attempting to strike a balance between patient access to innovative treatments and overall affordability. Payers and employers are evaluating how traditional specialty pharmacy management strategies and innovative models can support continued coverage of orphan drugs.

OBJECTIVE: To understand how health care stakeholders are meeting the financial challenges posed by the increasing number and cost of orphan drugs and how these strategies are affecting orphan drug acquisition for providers.
METHODS: A survey was conducted with payer, provider, and employer decision makers recruited from both AMCP and a proprietary database of market-access decision makers in July and August 2020. Respondents were asked about their experiences and activities in the orphan disease space, including tactics to manage affordability of drugs to treat orphan diseases.

RESULTS: Reinsurance was the most commonly utilized strategy to maintain affordability of the benefit for both payers (42\%) and employers (55\%). Although $31 \%$ of payers have adopted gene therapy carve-outs, no employers had done so. Approximately three quarters $(76 \%)$ of payers believe that limited distribution networks impede their abilities to manage orphan

\section{Author affiliations}

Erin Lopata, PharmD, MPH; Christopher Terrone, MS; and Ami Gopalan, PharmD, MBA, FAMCP, PRECISIONvalue, Chicago, IL. Nicholas Ladikos, PharmD, BCPS, BCGP, BCIDP, and Terry Richardson, PharmD, BCACP, Academy of Managed Care Pharmacy, Alexandria, VA.

\section{AUTHOR CORRESPONDENCE:}

Erin Lopata, 412.583.8953;

erin.lopata@precisionvh.com

J Manag Care Spec Pharm. 2021;27(6):706-13

Copyright $(2021$, Academy of Managed Care Pharmacy. All rights reserved.

drugs, compared with $4 \%$ who believe limited networks improve orphan drug management. For most payers (78\%), the decision to cover orphan drugs on either the medical or pharmacy benefit depends on the specific drug. Medical benefit coverage was driven primarily by site-of-care policies (55\%) and the lower drug cost of average sales price pricing (50\%). Pharmacy benefit coverage was driven primarily by a greater ability to manage the orphan drug ( $71 \%$ ) and by rebates ( $62 \%)$. One in $3(33 \%)$ of providers with experience treating orphan diseases acquire orphan drugs exclusively through buy and bill, whereas $10 \%$ acquire them exclusively through a specialty pharmacy provider. Buy-and-bill acquisition by providers was driven primarily by improved patient affordability (47\%) and 340 b pricing ( $47 \%)$. Specialty pharmacy 
provider acquisition was driven primarily by payer requirements $(64 \%)$ and reduced administrative burden (64\%).

CONCLUSIONS: Payers and employers are adopting innovative benefit designs and strategies to cover orphan drugs while maintaining plan affordability. Cost considerations are prominent factors in determining whether orphan drugs will be covered under the pharmacy or medical benefit and how providers will acquire orphan drugs.

Orphan drugs have been developed to address rare conditions affecting fewer than 200,000 patients in the United States. The number of products approved to treat orphan diseases has grown significantly in the past decade: in 2019, $44 \%$ of the U.S. Food and Drug Administration's 48 new drug approvals were for orphan diseases., ${ }^{1,2}$ With a robust pipeline of future orphan drugs and new indications for existing products, this trend is expected to continue. Although these innovations address conditions with limited or no treatment options, they often come with a significant cost: the average cost of an orphan drug is 4.5 times higher than the average cost of a nonorphan drug. ${ }^{3}$ As a result, spending on orphan drugs grew to account for $10 \%$ of overall drug spending in 2017, from 4\% in $1997 .{ }^{1}$ With orphan conditions by definition affecting a small number of patients in a population, payers have generally been able to absorb the high cost of the treatment; however, the cost burden of orphan disease treatments in aggregate may be reaching a tipping point that overwhelms the budgets of payers and employers. ${ }^{4}$

Health care payers are tasked with balancing affordability and patient access to an increasing number of innovative, high-cost therapies. Many payers have applied traditional utilization management strategies, such as prior authorization requirements, step therapy, and quantity limits, to manage orphan drugs. ${ }^{5}$ Even with effective utilization management techniques, payers must explore additional strategies to support ongoing affordability of these products, including innovative payment models and benefit design changes. Although self-administered orphan drug products are typically covered under the pharmacy benefit, products that are administered by a health care professional have the potential to be covered under either the pharmacy or medical benefit, which can have implications for both providers and patients. ${ }^{6}$ Aside from strategies that affect use of orphan drugs, both payers and employers have explored other approaches that affect how risk is spread across a population. These include use of reinsurance, which reduces the exposure of a payer or employer to catastrophic claims amounts, and use of separately funded high-risk pools for coverage for individuals who have high medical costs and complex medical conditions. ${ }^{7}$ Rare disease specific carve-out arrangements are an emerging strategy to protect payers and employers by contracting with a third party that assumes the risk for reimbursement, while also supporting coordination for patients and providers throughout the process. ${ }^{8}$

To better understand how payer, employer, and provider stakeholders are responding to the financial challenges associated with orphan drugs and how benefit design is affecting patient access, a survey was deployed to understand current and future strategies and perceptions. The results of this survey were discussed during a Partnership Forum that AMCP convened in September 2020 with various stakeholders to develop solutions and recommendations for addressing identified challenges and barriers to help support patient access to rare disease treatments. ${ }^{9}$

\section{Methods}

Participants were recruited via email from a proprietary database developed and owned by PRECISIONvalue, consisting of approximately 6,700 managed care decision makers and health care stakeholders and from among the AMCP Partnership Forum participants. The survey was fielded using the Qualtrics platform from July 16, 2020, to August 4, 2020.

Survey questions were developed following consultation with AMCP to identify critical issues in the orphan drug marketplace. The survey consisted of a total of 35 questions, with branching logic to direct segment-specific questions to payers, providers, and employers. For participants recruited from the PRECISIONvalue database, information about each participant's organization type, role, practice specialty (for providers), covered lives (for payers), and employees (for employers) was available from the database. The survey was double blinded, and participation was voluntary. Survey respondents received an honorarium for participation.

In the survey preamble, "orphan disease" was defined for participants as "a condition that affects fewer than 200,000 people in the United States." The survey consisted of questions to assess the decision-making process in the orphan disease space, economic strategies to pay for orphan drugs, the effect of benefit design on orphan drug management, and support for patients with orphan diseases. The current paper presents results from the questions focused on economic strategies for managing orphan drugs. The full survey instrument is available from the authors on request. 


\section{Results}

\section{RESPONDENTS AND DEMOGRAPHICS}

Fifty respondents recruited from the PRECISIONvalue proprietary database and 8 respondents recruited from the AMCP membership completed the survey. Demographic information beyond payer, provider, and employer segments are only reported for respondents recruited from the PRECISIONvalue database. Twenty-six respondents represented payer organizations, which included national, regional, local, Medicaid, and Medicare plans, as well as the payer components of integrated delivery systems, and pharmacy benefit managers. Thirteen payer respondents were pharmacy directors, and 7 were medical directors. The provider segment comprised 21 respondents with experience with rare disease patients and drugs, representing 13 specialties. Eleven respondents represented employers, including 3 employee benefit consultants and 2 coalitions. Covered lives and employee data were available for health plan and employer respondents recruited from the PRECISIONvalue database. Health plan respondents recruited from the database represented a total of 76 million covered lives. Employer respondents recruited from the database represented 556,000 total employees, whereas coalitions represented nearly 300 organizations with more than 1 million covered lives, and employee benefit consultants represented clients serving more than 20 million covered lives.

\section{STRATEGIES TO MAINTAIN PLAN AFFORDABILITY}

Payers and employers assessed 8 strategies to maintain plan affordability as additional orphan products become available. Respondents were asked to indicate whether each strategy was currently in use, planned for short-term implementation (within the next 18 months), for long-term implementation (beyond 18 months), or whether there were no plans to implement the strategy. Across both payers and employers, reinsurance was the most commonly utilized strategy to maintain affordability of the benefit, with $42 \%$ of payers and $55 \%$ of employers currently having a reinsurance strategy in place. Approximately one third of payers and employers (31\% and 36\%, respectively) had no plans to implement a reinsurance strategy. A gene therapy carveout strategy is utilized by $31 \%$ of payers currently, and up to two thirds of payer respondents expect to adopt such a strategy in the long term. In contrast, no employers currently indicated having a gene therapy carve-out in place or planned within the next 18 months, and only $18 \%$ plan to implement such a plan in the long term. Employers' second most common strategy for maintaining affordability of the benefit is shifting coverage of orphan drugs from the medical benefit to the pharmacy benefit, which was noted to be in place by $36 \%$ of respondents, with an additional $9 \%$ planning to implement in the long term. Other strategies assessed included an orphan drug carve-out, benefit exclusion, risk pools, an amortization or installment model, or a third-party subscription model, as shown in Figure 1.

Payers and employers were also asked to rate each strategy based on their perceived level of effectiveness in addressing affordability concerns. Payers rated benefit exclusion, gene therapy carve-out, and reinsurance to be the most effective; whereas employers rated third-party subscription (per member per month [PMPM]) model, reinsurance, benefit exclusion, and risk pools as most effective. Both payers and employers ranked shifting coverage from the medical benefit to the pharmacy benefit as the lowest in terms of perceived effectiveness. See Figure 2 for more details.

\section{ROLE OF LIMITED DISTRIBUTION NETWORKS}

Payers were asked how limited distribution networks affect their ability to manage orphan drugs. More than three fourths $(76 \%)$ of respondents indicated that limited distribution networks impede management, with only $4 \%$ indicating that they improve management. Respondents were given the opportunity to provide a free-text response to explain their rationale. An individual from a pharmacy benefit manager noted that a limited distribution network improves management by mandating where an orphan drug can be dispensed, which simplifies the process by negating the need to aggressively manage discounts. Alternatively, another respondent noted that a limited distribution network lacks transparency to find patients, access data, or follow endpoints for study. An individual from a payer component of a health system cited limited drug and provider access, inflated pricing, and suppressed competition as concerns associated with limited distribution networks.

\section{MEDICAL VERSUS PHARMACY BENEFIT FOR HEALTH CARE PROVIDER-ADMINISTERED PRODUCTS}

For the coverage of health care provider (HCP)-administered products, the majority of payers surveyed (78\%) stated that the decision to cover on the pharmacy or medical benefit is product dependent. Thirteen percent always cover on the pharmacy benefit using a specialty pharmacy, and $9 \%$ always cover on the medical benefit. Roughly half (52\%) of payer participants have site-of-care policies or strategies that affect orphan drugs; $32 \%$ have site-of-care policies or strategies in place but do not apply them to orphan drugs; and $16 \%$ reported having no site-of-care policies or strategies. Payers were asked to identify the specific reasons for directing coverage of $\mathrm{HCP}$-administered orphan drugs to 


\section{FIGURE 1 Payer and Employer Strategies to Maintain Affordability for Orphan Drugs}

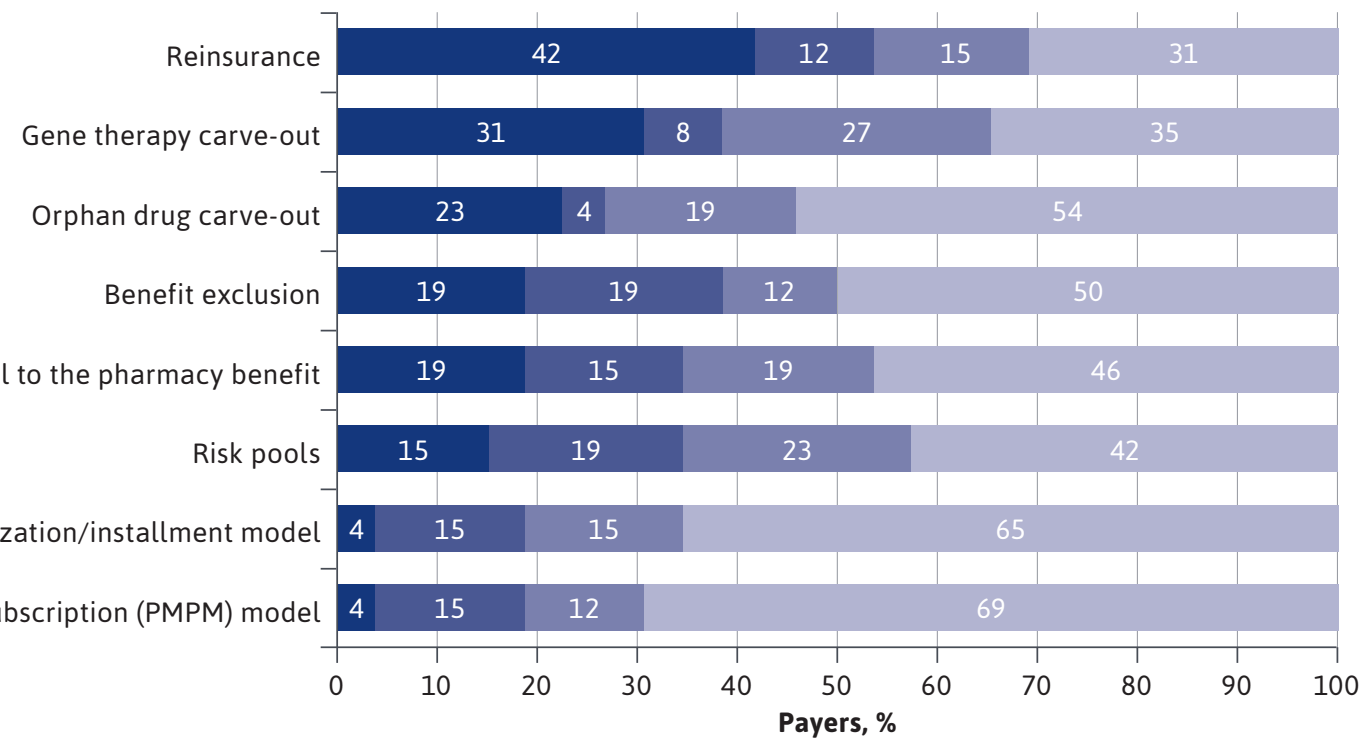

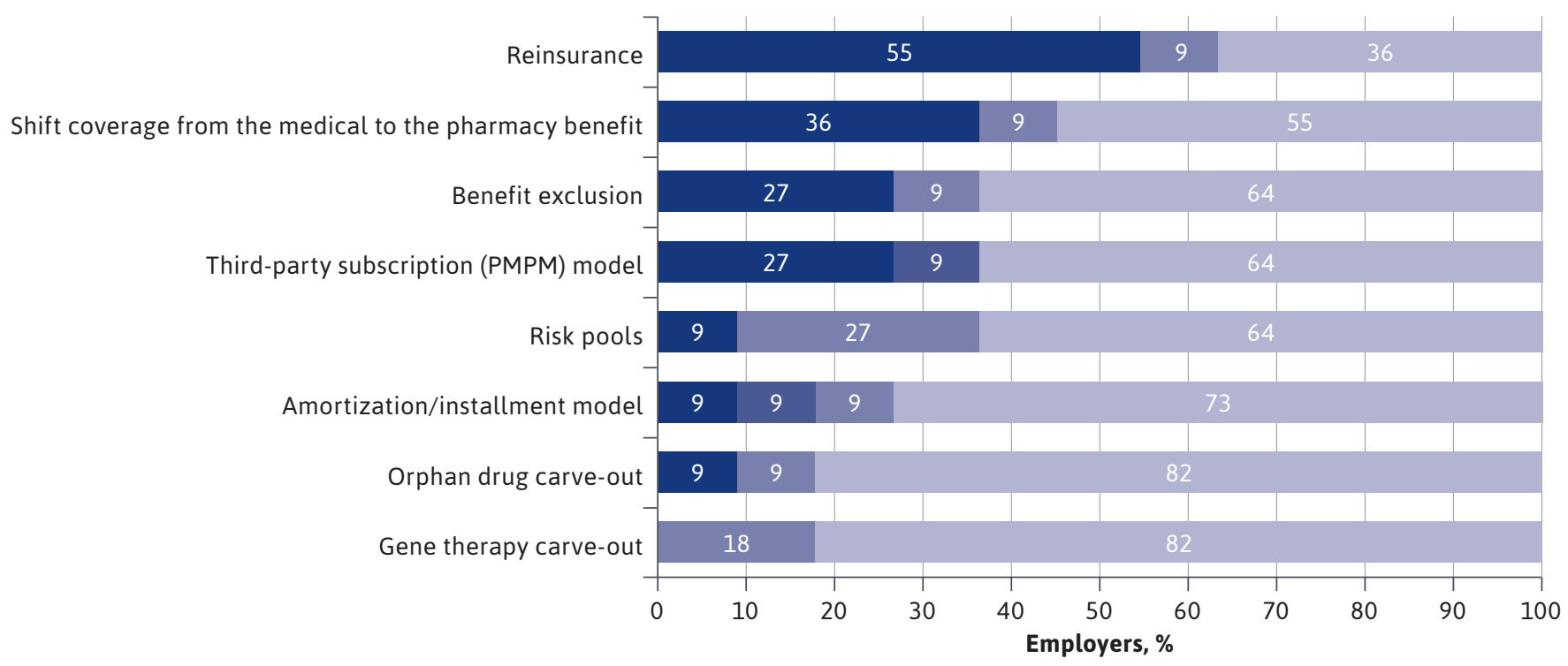

Note: Payers $(n=26)$ and employers $(n=11)$ were asked if their organizations currently use or plan to use any of the following strategies to maintain affordability as more orphan products become available.

PMPM = per member per month. 


\section{FIGURE 2 Payer and Employer Perceptions of Effectiveness for Strategies Addressing Affordability of Orphan Drugs}

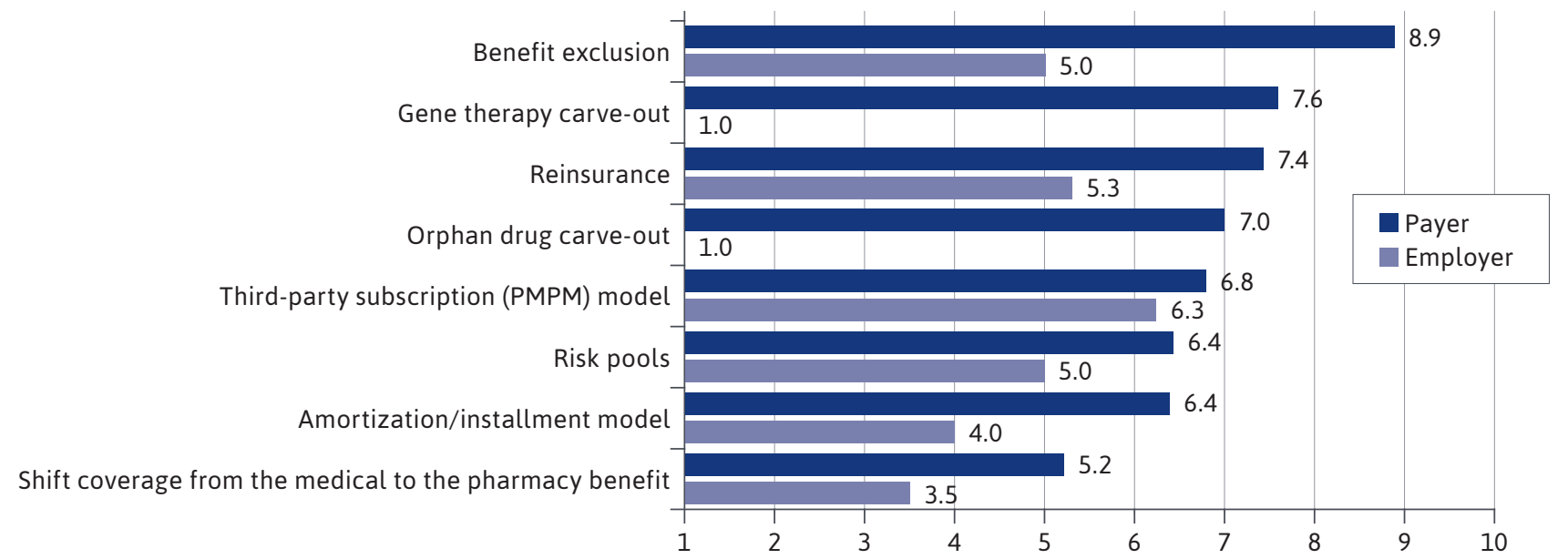

Note: Payers $(n=26)$ and employers $(n=11)$ were asked to rate the effectiveness of affordability strategies on a scale of 1 to 10 , with 1 representing a low level of effectiveness and 10 representing a high level of effectiveness.

$P M P M=$ per member per month.

the medical or pharmacy benefit. The top factors that drove payer coverage of $\mathrm{HCP}$-administered orphan drugs to the medical benefit were site-of-care policies (55\%), lowerdrug cost through average sales price (ASP) pricing (50\%), preference of network providers (35\%), and patient affordability (20\%). The top factors that drove payer coverage of $\mathrm{HCP}$-administered products to the pharmacy benefit were improved ability to manage product (71\%), rebates (62\%), ability to leverage resources from specialty pharmacy providers (SPP; 57\%), site-of-care policies (52\%), improved data granularity (38\%), preference of network providers $(38 \%)$, and patient affordability (24\%; Figure 3$)$.

Providers with experience treating patients with rare disease were also surveyed on their experiences and preferences related to orphan drug product access. The majority of providers obtain orphan drugs through the buy-and-bill process, with $33 \%$ reporting exclusive use of buy and bill and $38 \%$ obtaining orphan drugs mostly through buy and bill and sometimes through an SPP (Figure 4). Exclusive SPP use was reported by $10 \%$ of surveyed providers, and $15 \%$ reported mostly using SPP and sometimes obtaining through buy and bill. Providers were asked to identify the factors that drove their use of buy and bill or SPP for acquisition of orphan drugs. The top factors that drove providers to utilize buy and bill for orphan drug acquisition were improved patient affordability (47\%), 340b pricing (47\%), revenue generation (37\%), payer requirement for buy and bill (26\%), and less-aggressive payer management (21\%). The top factors that drove providers to utilize an SPP for acquisition of orphan drugs were payer requirement to use SPP (64\%), reduced administrative burden in managing inventory and billing payers (64\%), improved patient affordability (57\%), and SPP patient support services (36\%).

\section{Discussion}

The results of this survey underscore the effect of the increasing cost and number of orphan drugs on a broad group of stakeholders. Payer, employer, and provider stakeholders are reacting to these challenges and evolving their approach to meet their ongoing financial goals as well as the needs of patients with orphan conditions. Although each stakeholder has their own priorities related to use of drugs for the treatment of rare conditions, there are inherent interdependencies among these entities that can be leveraged to support shared goals and, ultimately, patient outcomes.

The robust pipeline of orphan drugs makes continued affordability of the benefit a top concern to both payer and employer stakeholders. Payer respondents are evaluating and, in some cases, starting to implement emerging strategies such as gene therapy or orphan drug carveouts, whereas employer respondents continue to focus on 


\section{FIGURE 3 Payer Drivers to Cover HCP-Administered Orphan Drugs on the Pharmacy Benefit}

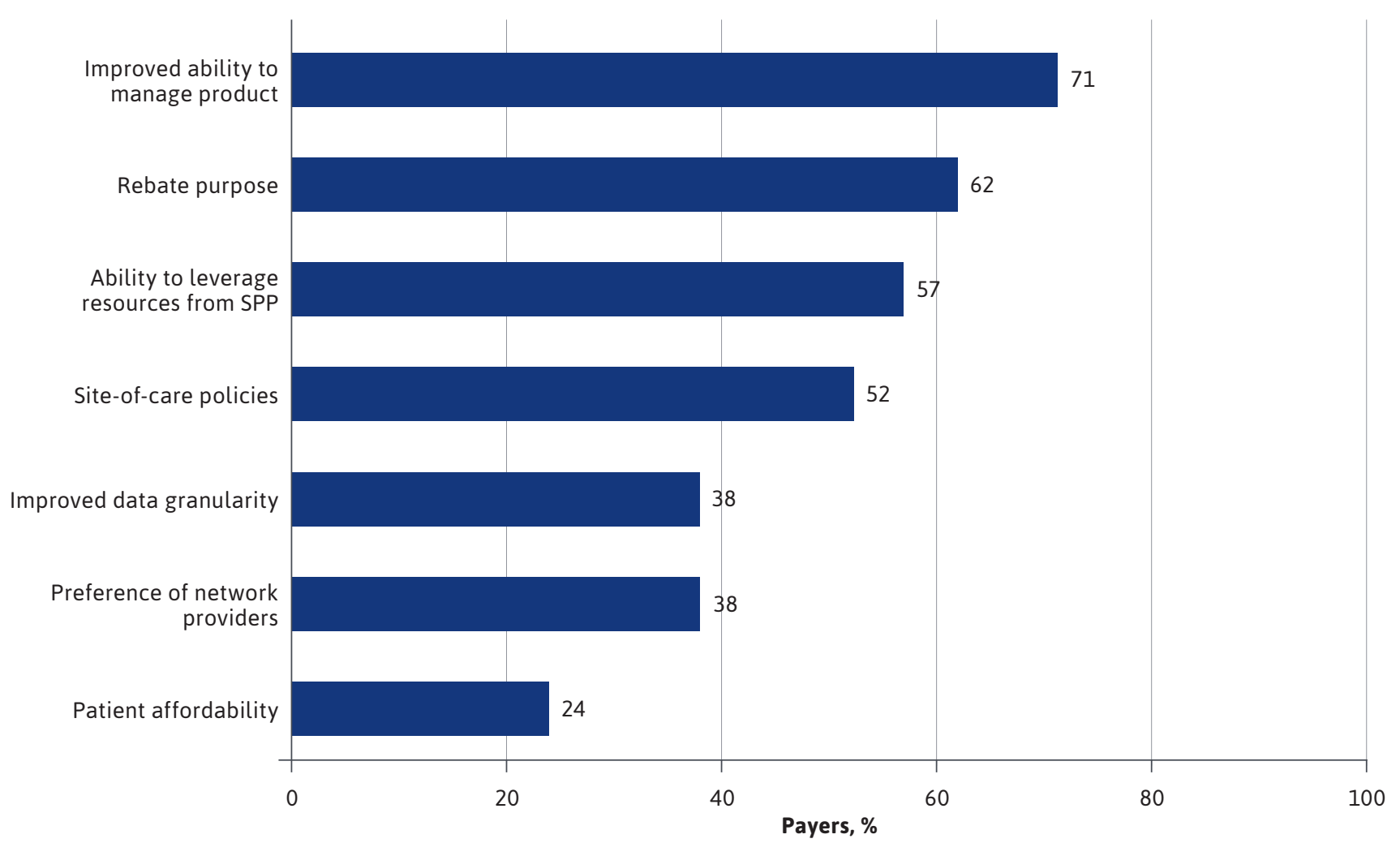

Note: Payers $(n=21)$ were asked to identify the reasons for preferring coverage of HCP-administered orphan products on the pharmacy benefit. Respondents could select more than 1 option.

$H C P=$ health care provider; SPP = specialty pharmacy provider

established specialty drug management strategies, such as shifting coverage from the medical to the pharmacy benefit. This is likely due to employers relying on their health plan partners to recommend innovative strategies. Our results were consistent with the 2021 Business Group on Health survey that found that over the next 2-3 years, $37 \%$ of employers surveyed plan to purchase stop-loss insurance, $21 \%$ are considering risk pools, and $9 \%$ are considering a drug financing model such as amortization to pay for high-cost drugs. ${ }^{10}$ Further research is needed to clarify why payers appear to be early adopters of some innovative strategies and to gauge whether employer implementation will eventually match that of payers. The survey results also indicated that benefit exclusion is, or will be, a commonly utilized strategy across payer and employer respondents, which could affect patient access to rare disease drugs. Limited distribution networks, while noted to impede management of orphan drugs by payers, will likely continue to be a strategy used to support effective product allocation, as it is purported to help ensure the safe distribution of high-risk/high-cost drugs to small patient populations. In addition, limited distribution networks may offer the specialized management and care coordination patients with rare diseases often require.

Our survey found that many of the payers surveyed are considering coverage of HCP-administered orphan drugs on the pharmacy benefit, primarily to achieve better management of the product through utilization-management programs. Although somewhat contradictory, both payers and employers surveyed believe that shifting orphan drugs to the pharmacy benefit from the medical benefit was only a moderately effective strategy to manage the cost of these products. Site-of-care policies and strategies that seek to promote patients receiving infused drugs in lower-cost 


\section{FIGURE 4 Provider Use of Buy and Bill and Specialty Pharmacy Provider to Obtain HCP-Administered Orphan Drugs}

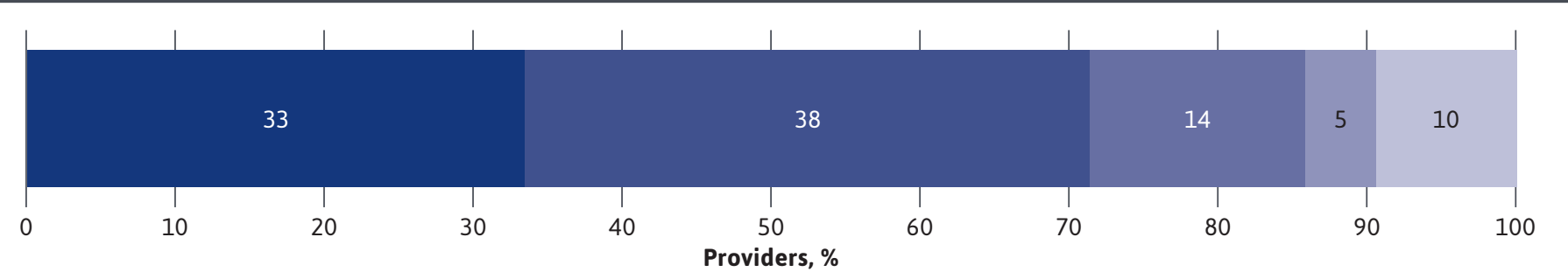

Providers, \%

$\begin{array}{ll}\text { Always buy and bill } & \text { Mostly through SPP, sometimes buy and bill } \\ \text { Mostly buy and bill sometimes through SPP } & \text { Always obtain from SPP } \\ \text { Equal frequency of buy and bill and obtaining from SPP } & \end{array}$

Note: Providers $(n=21)$ were asked how their organizations typically acquire orphan products.

$\mathrm{SPP}=$ specialty pharmacy provider.

alternate locations (e.g., physician office, infusion suite, home) were significant factors driving the decision to cover orphan drugs on either the pharmacy or medical benefit. Although patient cost-share requirements can differ between the pharmacy and medical benefits, patient affordability was not high in the considerations ranked by payers. Additional research is needed to assess how coverage of orphan drugs on either the pharmacy or medical benefit affects patient adherence, persistence, clinical outcomes, and total cost of care in the long term.

Providers who manage patients with orphan conditions were also surveyed as part of this research. In contrast to payers, the providers surveyed were more likely to consider the effect of pharmacy or medical benefit utilization on patient affordability. Payer mandate was the top driver of providers utilizing an SPP to obtain an HCP-administered orphan drug; however, many providers also noted the advantages of using an SPP, including reduced administrative burden and availability of SPP resources for their patients. There may be other factors not evaluated by this research that may influence provider perception around the benefit of SPP use for HCP-administered orphan drugs, such as the size of the provider's practice, staff and resources available, and whether the provider is affiliated with a health system.

Although this survey captured broad trends across a diverse group of stakeholders, there would be value in delving into the reasoning behind many of these responses in future qualitative research. Additional research with an increased sample size could also potentially identify further trends and perspectives to explore. Additionally, this survey focused on financial and benefit considerations across orphan drugs, and we recognize that there may be nuances based on the specific drug or therapeutic area that would be worth exploring further. Finally, as this remains a rapidly evolving area, additional research may describe how the uptake of these practices by payers influences uptake by employer and affects other stakeholders, such as providers and patients.

\section{LIMITATIONS}

Several limitations apply to our findings. First, these findings are based on a small sample size. Orphan diseases are by definition rare, resulting in a narrower field of knowledgeable respondents compared with more prevalent conditions such as hypertension, diabetes, or lung cancer. Although payer respondents in this sample represented more than 70 million covered lives, the employer segment represented a smaller pool of employers. While coalitions and employee benefit consultants represent a broad employee population across geographic regions, respondents from employer coalitions and employee benefit consultants may not reflect the opinions of all individual employer members. The number of patients under the care of our provider respondents is not known. Because of the small sample size, comparisons among payer, provider, and employer segments are not statistically significant, and intragroup differences (e.g., between regional and national plans) may have an outsized effect on the aggregated results.

Second, participation bias may drive individuals with greater interest or knowledge in the survey topic to respond disproportionately. 
Third, the survey consisted mostly of closed-ended questions with prepopulated options. Because few questions contained an open-ended component and most did not include options for "other" or "none of the above," our findings might not have captured emerging or infrequently encountered trends.

\section{Conclusions}

Payers and employers are turning to innovative management strategies to offer coverage of orphan drugs while maintaining plan affordability. These strategies are considered at least as effective as traditional management tools, and broader implementation is expected over the next 18 months. Payers are using the decision to cover orphan drugs under either the medical or pharmacy benefit as a cost-control lever, and providers are weighing patient affordability in their decision to acquire orphan drugs via buy and bill. It will be important for all stakeholders in the orphan disease space to be familiar with these trends, as they affect not only the payers, providers, and employers surveyed here but also patients with rare diseases and pharmaceutical manufacturers.

\section{DISCLOSURES}

This research was sponsored by AMCP and PRECISIONvalue. Lopata, Terrone, and Gopalan are employees of PRECISIONvalue. Ladikos and Richardson are employees of AMPC. The authors have nothing further to disclose.

This research was presented during the AMCP Partnership Forum "Preparing for and Managing Rare Diseases" held virtually September 8-10, 2020.

\section{REFERENCES}

1. IQVIA Institute for Human Data Science. Orphan drugs in the United States: growth trends in rare disease treatments. October 2018. Accessed January 24, 2021. https://www.iqvia.com/-/media/ iqvia/pdfs/institute-reports/orphandrugs-in-the-united-states-growthtrends-in-rare-disease-treatments. pdf?\& $=1605578897396$

2. U.S. Food and Drug Administration. New drug therapy approvals 2019. January 6, 2020. Accessed January 24, 2021. www.fda.gov/drugs/new-drugsfda-cders-new-molecular-entities-andnew-therapeutic-biological-products/ new-drug-therapy-approvals-2019

3. EvaluatePharma. Orphan drug report 2019. 6th ed. April 2019. Accessed January 24, 2021. https://info.evaluate.com/rs/607-YGS-364/images/ EvaluatePharma\%20Orphan\%20Drug\%20 Report\%202019.pdf

4. Tozi J. Gene therapy drugs that cost millions have employers and health plans worried. Insurance J. September 3, 2019. Accessed January 24, 2021. https:// www.insurancejournal.com/news/ national/2019/09/13/539591.htm

5. Chambers JD, Panzer AD, Kim DS, Margaretos NM, Neuman PJ. Variation in US private health plan's coverage of orphan drugs. Am J Manag Care. 2019;25(10):508-12.

6. Hyde R, Dobrovolny D. Orphan drug pricing and payer management in the United States: are we approaching a tipping point? Am Health Drug Benefits. 2010;3(1):15-23.
7. Busch F, Houchens R. Reinsurance and high-risk pools: past, present, and future role in the individual health insurance market. June 6, 2017. Accessed January 24, 2021. https://us.milliman.com/en/ insight/reinsurance-and-highrisk-poolspast-present-and-future-role-in-theindividual-health-i\#

8. Jackson A, Naber J, Runyan B. Exploring the benefits of a multiyear payment arrangements for self-insured employers and TPAs: partnering with manufacturers of gene and cell therapies. January 2020. Accessed January 24, 2021. https://milliman-cdn.azureedge. net/-/media/milliman/pdfs/articles/ exploring_-the_benefits_of_multiyear_payment_arrangements_for_selfinsured employers and tpas.ashx

9. AMCP Partnership Forum. Preparing for and managing rare diseases. J Manag Care Spec Pharm. Published online February 2, 2021. Accessed February 8, 2021. https:// www.jmcp.org/doi/pdf/10.18553/ jmcp.2021.20546

10. Business Group on Health. 2021 large employers' health care strategy and plan design survey. August 2020. Accessed January 24, 2021. https:// www.businessgrouphealth.org/ resources/2021-large-employers-healthcare-strategy-and-plan-design-survey 